Abstracts of

34th Health Topics for

Tokyoites

Juntendo Medical Journal

2014. $60(5), 467$

\title{
Immunization in Childhood in Japan: the Past, the Present, and the Future**
}

\author{
KEN HISATA* \\ * Department of Pediatrics, Juntendo University Faculty of Medicine, Tokyo, Japan
}

In the 1970-80s, Japan developed various vaccines ahead of the rest of the world. DPT vaccine (diphtheria, tetanus, and pertussis) and Japanese encephalitis vaccine were introduced abroad from Japan. In addition, the Japanese Oka strain used for varicella vaccine is the only strain recommended by the World Health Organization (WHO). However, problems related to vaccination such as aseptic meningitis with MMR (measles-mumps-rubella vaccine) subsequently occurred. As a result, in Japan in the 1990s, new vaccines were not developed and vaccination policy stagnated. On the other hand, many countries around the world promoted vaccination policies from the viewpoint of preventive medicine and developed various vaccines that were incorporated into routine immunization. However, the vaccine gap in Japan has begun to improve recently. Specifically, vaccines that are recommended increased in number and the immunization schedule became complicated. In terms of the past and present vaccination policies in Japan, this session gives an outline of the effects of vaccines and issues regarding vaccines and immunization schedules. Unfortunately, rubella was prevalent in Japan last year, and cases of congenital rubella syndrome have increased. Therefore, we must provide education on the importance of vaccination and herd immunity in Japanese vaccination policy.

Key words: pediatrics, vaccination, schedule, herd immunity

\footnotetext{
Ken Hisata

* Department of Pediatrics, Juntendo University Faculty of Medicine 2-1-1 Hongo, Bunkyo-ku, Tokyo 113-8421, Japan

TEL: + 81-3-3813-3111 (Ex 5670) E-mail: ken-hisa@juntendo.ac.jp

**34th Health Topics for Tokyoites: The Current Vaccine Situation〔Held on June 21, 2014〕

〔Received Sep. 4, 2014]
} 